\title{
Human cerebral organoids and neural 3D tissues in basic research, and their application to study neurological diseases
}

\author{
Theresa S P Rothenbücher*,1 \& Alberto Martínez-Serrano*,1 \\ ${ }^{1}$ Department of Molecular Biology, Univ. Autónoma de Madrid; \& Department of Molecular Neuropathology, Center of Molecular \\ Biology Severo Ochoa (CBMSO, UAM-CSIC), Nicolás Cabrera 1, 28049 Madrid, Spain \\ *Authors for correspondence: amserrano@cbm.csic.es
}

First draft submitted: 14 December 2018; Accepted for publication: 3 January 2019; Published online: 25 January 2019

Keywords: 3D tissues $\bullet$ cerebral organoids $\bullet$ human pluripotent stem cells $\bullet$ neurological disease $\bullet$ tissue patterning - tissue scaffolding

Research with neural tissue in 3D is receiving great attention recently, since 3D offers a more physiological setting than traditional $2 \mathrm{D}$ cultures. In this commentary we overview and compare cerebral organoids and other $3 \mathrm{D}$ aggregated cell cultures. Last we briefly summarize examples of neurological diseases, mostly neurodevelopmental, where organoids are starting to be of help to understand the disease etiology, and in some cases to design therapies.

In vitro studies of neural tissue development, physiology, biochemistry, and molecular and cell biology are presently understood to be more relevant if experimental procedures are conducted in $3 \mathrm{D}$ as compared with $2 \mathrm{D}$ (monolayer cultures). The rationale behind this notion resides in the mutual interaction of cells in $3 \mathrm{D}$, the possibility for the cells/tissue to generate its own extracellular matrix, and the cells capacity to establish 3D projections and multiple interactions, and that this would yield tissue constructs and organoids that better resemble the in vivo developmental processes, and the organization of functional, mature tissues, as well as the physiology and pathophysiology of neurodevelopmental processes, possibly including aging.

The 3D human neural tissue culture dates back to decades ago, early 1990s, when Weiss and collaborators described the generation of human neurospheres (spheroids), comprised of human neural stem cells, co-existing with differentiated cells [1]. The neurosphere culture method rapidly expanded and was extensively used, until presently. Interestingly, by that time and for many years, the focus was on how to proliferate the recently identified neural stem cells, rather than on the cytoarchitecture of the (putatively) self-organizing neural tissues in neurospheres. As a matter of interest, at that time the term 'neural stem' was not even widely accepted and therefore it was important to demonstrate their successful proliferation in culture. Consequently, neurosphere cultures were rendered as an experimental tool to proliferate the few neural stem cells that remained in such stem cell stage. Later on, the capacity to generate neurospheres, was used in clonogenic assays, to identify neural stem cells in a given neural cell preparation. But little, if any, attention was paid to the 3D organization of developing neural tissue in the 3D neurospheres.

With the availability of pluripotent stem cells (PSCs) by the end of the 90s, a renewed interest in 3D cultures arose. The trigger for this revival was the PSC' characteristic of pluripotency (understood as the capacity to give rise to cell derivatives of different embryonic germinal layers, that is, endoderm, mesoderm and ectoderm). The new angle was to let the cells develop into different tissues, after providing them with the appropriate developmental signals. Subsequently, during the last decade, different groups managed the development of organ-like tissues in 3D cultures of PSCs, that resembled some of the histological features, tissue architecture and some basic functionality of their in vivo counterparts (e.g., intestine, retina, cerebral cortex, ...; [2-5]).

More recently, we have seen an exponential rise of published studies using 3D human neural cell cultures, ranging from simple cell aggregates (lacking tissue cytoarchitecture, cellular self-organization), to other rather organized tissues generated from embryoid bodies. It is important to highlight at this point that organoids are much more complex than simple 3D aggregates of cells. An organoid is generated through a series of complex developmental decisions and continuous processes, combined with a progressive restriction of proliferation of the 
progenitors responsible for the generation of such organ-like structure. Therefore, the successful in vitro organ-like development is the result of a highly complex-combinatorial, time-restricted contribution of signals and events. In other words, it is the re-action of the developing tissue to the provided cues, resulting into decreased proliferation while simultaneously and progressively integrating developmental signals, which prompt migration, differentiation and maturation that together render an organ-like histological structure. All of these processes are similar to the events during embryonic development in vivo. The 3D cellular aggregates, on the other hand, are more simple to generate and less likely to integrate any development signals or to show cellular self-organization. However, they constitute good models for the quick assessment of cell biology in 3D (see an example on Alzheimer's disease, below).

This is what we can conclude from our own work with both types of 3D tissues (Supplementary Figure 1), where we have compared 3D cell aggregates with real cerebral organoids, finding that their properties are essentially different, and therefore, their potential uses become different. In brief, real organoids recapitulate development and maturation, possibly aging, and are therefore useful to study biological processes related to these events, whereas $3 \mathrm{D}$ aggregates provide simple means to evaluate very basic questions in $3 \mathrm{D}$, like neuron generation or neuron/glia differentiation and survival, constituting a good instrumental tool, but not recapitulating nerve tissue development.

Once the difference between cell aggregates and organoids has been discussed, we will explore the potential that the currently available cerebral organoid models offer for the investigation of neurological diseases, deficits, and also for basic neurobiology research.

\section{Origins of the cerebral organoid technology}

After seminal studies by the groups of Sasai, Clevers, Knoblich and Pasca [2-5], the available technical knowledge was coaxed into published procedures for the generation of differentiated neural/neuronal clusters or neuronal regions in $3 \mathrm{D}$ in cerebral organoids [5-7]. These $3 \mathrm{D}$ tissue constructs showed some regions containing neural tissue with a cytoarchitecture reminiscent of their in vivo counterparts (mostly cortical tissue, since this is the default phenotype according to those protocols). Therefore, those 3D tissues were described as 'cerebral organoids' or 'cerebroids' (colloquially referred to as 'mini-brains'). However, the term mini-brain is profoundly misleading, since these 3D tissues do not organize themselves into discrete regions showing interconnections resembling the developing brain.

When examining the histology of the cerebral organoids in detail, the outcome was on the one hand new and exciting, thus promising. But on the other hand, it became clear that the current cerebroids have limitations and there still remain many challenges to overcome before having a real human brain model. Multiple reports described the appearance of neuralized tissue, with some cellular organization, but the whole composition of such 3D tissue constructs did not resemble or was not reminiscent of a developing brain. In contrast, the appearance of discrete, regionally specified brain regions was very limited, and subjected to a stochastic process. In some organoids there were neural tissues, while in others the neural tissue did not appear. In some successful, neuralized, cerebral organoids, some markers of regionally specified neurons appeared, randomly and without control by the investigator. In some cases, groups of cells expressing cortical or hypothalamic neurons appeared, while in many other cases those regions did not appear $[2,5,7,8]$. This scenario calls for a better engineering by the experts. Engineers collaborating with biologists. Recently, a simple scaffolding procedure (randomly arranged, floating poly(lactide-co-glycolide) fibers to which the embryoid bodies attached) has been described, partially enhancing the reproducibility of generating successful cerebroids containing cortex-like tissue from human PSCs [8].

Essentially, we are at a very initial stage in the research process to decipher how to generate cerebral organoids really recapitulating the development of the human brain. For this challenging task, it is obvious that some type of scaffolding, providing regional and time-resolved developmental cues, physical support, together with an artificial or biological vascularization/perfusion, is needed. For some applications, the external provision of microglial cells will definitely be a must, when dealing with physiopathological processes mediated by microglia. It is unclear at the moment how to combine different cell types during the generation of successfully developing cerebral organoids.

After recognizing the exceptional research opportunities that cerebroids offer, since they recapitulate the generation of some histologically organized brain tissue, we need to adopt a critical approach. Presently, a cerebral organoid is an almost spherical structure (isotropic, no orientation landmarks, no polarization or developmental axis), where different regions develop/appear randomly, with no recognizable (brain) organ-like patterns, showing only discrete regions resembling parts of the in vivo brain organ.

Initial attempts to provide the cerebroids with some kind of regional patterning have been reported (e.g., for midbrain, forebrain or hypothalamic identity [9-11]; or striatal and cortical [12]). Currently used procedures for 
cerebroid generation use culture media and inductive/differentiation/maturation cocktails optimized for their default differentiation toward the generation of cortical tissue. The studies aiming at generating other brain identities used procedures originally developed in $2 \mathrm{D}$ tissue culture to provide the cells with the appropriate, chronologically ordered, development cues, applied to the cells in the form of soluble factors/morphogens dissolved in the culture medium. These studies described a 'bulk' patterning, bathing the developing organoids with cocktails that thus convert the whole structure into, for example, hypothalamic or midbrain identity. This approach resulted in a tissue construct that did not recapitulate neither brain organ development, nor the brain circuitry. Regional induction existed, but it was not a true spatially resolved or organized patterning. The whole organoid became one or another identity. For developmental studies, an imaginative, elegant, alternative to this situation was to promote the fusion of differently patterned cerebroids, to study cell migration and the interconnections they could develop when becoming physically in contact [12-14]. Obviously, this was an instrumental strategy to get different regions (organoids) physically interacting, but distant from a truly spatially resolved patterning approach in a single organoid.

\section{Examples of diseases approached using the cerebroid technology}

In spite of technical hurdles and reproducibility related difficulties inherent to the cerebroid model system, researchers have successfully managed the study of the ethiology, as well as potential therapies, for diseases of high personal and social impact and burden.

Most investigations have used brain organoids to model neurodevelopmental diseases like microcephaly [5,15], autism [16,17], Rett syndrome [18], Miller-Dieker syndrome [19,20], schizophrenia [21], Sandhoff disease [22] or Zika virus (ZIKV) infection involving neurodevelopment [9,15,23-27]. Also neurodegenerative diseases like Alzheimer's disease (AD) [28] and the potential for modeling Parkinson's disease [11] were approached. Importantly, disorders that manifest during embryonic/fetal development are more suitable for modeling in brain organoids, since brain organoids, at the present stage, recreate particularly well the early brain development.

There are several methods available to obtain brain organoids recapitulating disease features. First, adult cells (e.g., skin) can be taken from patients and reprogrammed into induced PSCs (iPSCs). Furthermore, it is possible to introduce known mutations that are causally related of a disease into healthy cells. Also, in case of Zika virus for example, a viral infection of the organoid is performed to study modifications induced by the disease. In parallel to these disease models, organoids that were generated with healthy cell lines act as control for a comparison. Recent studies are briefly summarized below to illustrate how brain organoids may be useful tools in the field of neurological disorders.

In the case of microcephaly, Lancaster $e t$ al. were able to model some patterns of the disease. They observed premature neuronal differentiation that caused a reduction of early progenitors and led to an overall reduced size of the neuronal tissue compared with the (healthy) control organoids [29]. Gabriel et al. however, studied microcephaly, which was induced by Zika viral infection [15]. They observed very similar manifestations, which strengthen the reliability of modeling disease in brain organoids.

Several other studies investigated different strains of the Zika virus [9,15,23-27]. They all confirmed that a ZIKV infected brain organoid has a reduced size similar to microcephaly and shows increased apoptosis. Moreover, Li et al. highlight folding defects that were visible in ZIKV organoids [23], and Qian et al. reported a decreased neuronal layer thickness and an enlarged lumen [9]. Janssens et al. revealed that an African Zika strain modifies DNA methylation in developing neurons by disturbing the activity of DNA methyltransferase [27]. Yoon $e t$ al. identified the NS2A protein as responsible for reduced proliferation of radial glia cells in a Zika-infected brain by reducing adherens junctions [26]. Dang et al. found out that Zika virus activates the Toll-like receptor 3, which is part of the innate immune response, causing apoptosis and at the same time dysregulation of genes that steer neurogenesis, axon guidance and differentiation [25]. Taken together, these studies emphasize the utility of brain organoids to reveal new aspects of a human brain disease, which is the baseline on the way toward finding a therapy.

Mariani et al. studied glutamate neuron differentiation in brain organoids generated from cells of patients with autism spectrum disorder (ASD) [16]. They found, that in ASD the FOXG1 gene is strongly expressed, which leads to an overproduction of inhibitory neurons (GABA neurons). The amount of glutamate neurons however, was not increased, which causes an imbalance between excitation and inhibition. Mariani et al. concluded that the FOXG1 gene is thus a molecular signature of ASD and could represent a future target in ASD therapy. Wang et al. produced telencephalic organoids with a mutation in the CHD8 gene to study its impact in autism and schizophrenia [17]. They found that CHD 8 regulates the expression of the genes AUTS2 and TCF4 involved in ASD and schizophrenia, 
respectively. They highlight the possibility of identifying a drug target with the combined knowledge obtained from their and other studies.

Stachowiak et al. modeled schizophrenia with cerebroids and investigated the role of FGFR1 [21]. The patientderived organoids showed a very different architecture compared with the control. Among others they displayed an abnormal premature subcortical neurogenesis, which was apparent by a disruption of the developmental strata. Ki67 positive (cycling) cells showed an increased proliferation by migrating from the ventricular zone into the intermediate and cortical zone, which led to a reduction of mature neurons in the outer zone. They concluded that these cells have failed to exit the cell cycle and consequently to differentiate. Due to this, the cortical neuronal cells have a very low level of nuclear FGFR1, which is known to induce a dysregulation of downstream gene expression crucial for neurogenesis. This would lead to cortical malformation during brain development of schizophrenia patients. Stachowiak et al. state that changes in interneuron directionality could be the reason for disrupted cortical interactions in schizophrenia [21]. Complementary, Ye et al. discovered with their patient-derived schizophreniaforebrain organoids (in parallel to in vivo studies), that a C-terminal mutation of DISC1 disrupts the interaction with Ndel1 [30]. They ascertained that this leads to a dysregulation of neuronal stem cell population in the ventricular zone during forebrain development.

Raja et al. succeeded in modeling disease patterns of Alzheimer's - like amyloid aggregation, hyperphosphorylated tau protein and endosome abnormalities in cerebral organoids. Also, they successfully tested the efficacy of $\beta$ - and $\gamma$-secretase inhibitors to reduce amyloid and tau pathology in Alzheimer patient-derived brain organoids [28]. Interestingly, an alternative model did not use human PSCs, but a $v$-myc immortalized cell line of ventral mesencephalic origin (ReNcell VM), to generate 3D tissue constructs for modeling $\mathrm{AD}$ [31].

A step toward modeling Parkinson's disease in brain organoids was done by Monzel et al. [11] and Jo et al. [10], who were able to generate brain organoids with midbrain identity, which contain not only dopaminergic neurons, but also show electrophysiological activity and neuromelanin accumulation. It will be interesting in the future to understand how dopaminergic neurons develop, and age, in Parkinson's disease brain organoids.

All these studies show that brain organoids - even if the experimental model is far from being optimized have great potential for the study of some neurological diseases. Mostly, as described above, neurodevelopmental conditions lead to a disease. However, the usefulness of cerebral organoids for neurodegenerative conditions (e.g., AD, Parkinson's disease and others) faces many challenges. As a main challenge stands the translation of findings obtained in an in vitro system - cultured in vitro for many months - to reproduce the actual events occurring during the progression of a chronic degenerative disease in the human brain. Nevertheless, two aspects are of crucial importance: brain organoids from human PSCs provide the investigator with human tissues for research, and their development in 3D, expected to better recreate human brain biology. Especially important will be to exploit the use of brain organoids in the context of the 3Rs concept related to progressively reduce the use of animals in research. Human cerebral tissue will accelerate and foster successful research into the actual molecular and cellular key events leading to human disease, while reducing the unnecessary use of animals in research, a long time awaited goal in neurobiology research.

\section{Attempts to enhance reproducibility, \& improving the experimental model}

In order to develop improved cerebral organoids for neurobiology/neurological research, it needs to be solved how to prospectively, provide spatially resolved regional patterning and specification cues in a whole cerebroid. Optimally, neurological research needs of cerebroids in which discrete brain regions could be prospectively generated at desired spatial locations being meaningfully organized, spatially resolved, to study for instance how projection and communication between different developing regions are established, modulated, remodeled, and how they survive and degenerate.

One of the possible solutions to this hurdle will be to engineer scaffolds with biocompatible materials for the development of cerebroids with improved organization. Such scaffold should provide perfusion/vascularization, as well as spatially distinct developmental cues, and overall, allow for the development of a self-organizing cerebroid. Optimally, such scaffold should also have electrically conducting properties for the stimulation and monitoring (bio-sensing) of the developing cells.

Next, the use of genuine (self-organized, nonpatterned) cerebral organoids as a model system for any type of systematic study, is hampered from a very low reproducibility rate. As described above, the generation of neural tissue in a cerebral organoid occurs stochastically. Moreover, the different neuronal types in the same organoid are generated almost randomly and most importantly, without control by the investigator. However, since the 
development of an organoid resides in the self-organization and self-development capacities of the tissue, any interference with the 'self-drive' may result in an impaired development of the organoid, a difficulty that should be carefully considered.

While recognizing the interest of bulk-patterned organoids (very similar to patterned neurospheres) for some limited studies, the ideal scenario would be to generate an organoid with prospectively generated brain region identities at different locations, and that will show some type of communication/projection between different brain regions, spatially separated. For instance, to study cortico-striatal or nigro-striatal projections, and many others.

Basic requirements would be to enlarge the tissue constructs; to provide the developing organoid with a supportive (perfusable) scaffold, which would both provide physical and physiological support, and; providing spatially separated, regional developmental cues, for instance by hydrogels deposited at different locations in the scaffolds, loaded with different developmental cue molecules.

Research in this line includes attempts to provide the organoids with vascularization [32,33] and blood-brain barrier [34] in various ways. We expect seeing exciting developments in these research lines, which will help to exploit the full potential of cerebral organoids.

\section{Acknowledgements}

The authors would like to thank all members of the lab for their continued help and input, especially SG López and MP Pereira. The authors would also like to thank E Richard (CBMSO) for her help in the initial steps of their research project.

\section{Financial \& competing interests disclosure}

The present work on organoids and TSP Rothenbücher, is funded by an MSCA-ITN-ETN grant 722779, Training4CRM and by the Spanish Ministry of Science, Economy and Competitiveness, call Retos, Ref: SAF2017-83241-R. The authors have no other relevant affiliations or financial involvement with any organization or entity with a financial interest in or financial conflict with the subject matter or materials discussed in the manuscript apart from those disclosed.

No writing assistance was utilized in the production of this manuscript.

\section{Ethics statement}

The work developed in the research group was approved by the local UAM Ethics Committee, and authorized by the Local Government of Comunidad Autónoma de Madrid, Consejería de Sanidad, after a positive report by the 'Comisión de Garantías', from the Health Institute Carlos-III.

\section{Open access}

This work is licensed under the Creative Commons Attribution-NonCommercial-NoDerivatives 4.0 Unported License. To view a copy of this license, visit http://creativecommons.org/licenses/by-nc-nd/4.0/

\section{References}

1. Reynolds BA, Weiss S. Generation of neurons and astrocytes from isolated cells of the adult mammalian central nervous system. Science 255(5052), 1707-10 (1992).

2. Kadoshima T, Sakaguchi H, Nakano T et al. Self-organization of axial polarity, inside-out layer pattern, and species-specific progenitor dynamics in human ES cell-derived neocortex. Proc. Natl Acad. Sci. USA 110(50), 20284-20289 (2013).

3. Marton RM, Paşca SP. Neural differentiation in the third dimension: generating a human midbrain. Cell Stem Cell 19(2), 145-146 (2016).

4. Drost J, Artegiani B, Clevers H. The generation of organoids for studying Wnt signaling. Methods Mol. Biol. 1481, 141-59 (2016).

5. Lancaster MA, Renner M, Martin CA et al. Cerebral organoids model human brain development and microcephaly. Nature 501(7467), 373-379 (2013).

6. Lancaster MA, Knoblich JA. Organogenesis in a dish: modeling development and disease using organoid technologies. 345(6194), 1247125 (2014).

7. Sutcliffe M, Lancaster MA. A simple method of generating 3D brain organoids using standard laboratory equipment. Methods Mol. Biol. (2017).

8. Lancaster MA, Corsini NS, Wolfinger $S$ et al. Guided self-organization and cortical plate formation in human brain organoids. Nat. Biotechnol. 35(7), 659-666 (2017).

9. Qian X, Nguyen HN, Song MM et al. Brain-region-specific organoids using mini-bioreactors for modeling ZIKV exposure. Cell 165(5), 1238-1254 (2016). 
10. Jo J, Xiao Y, Sun AX et al. Midbrain-like organoids from human pluripotent stem cells contain functional dopaminergic and neuromelanin-producing neurons. Cell Stem Cell 19(2), 248-257 (2016).

11. Monzel AS, Smits LM, Hemmer K et al. Derivation of human midbrain-specific organoids from neuroepithelial stem cells. Stem Cell Reports 8(5), 1144-1154 (2017).

12. Bagley JA, Reumann D, Bian S, Lévi-Strauss J, Knoblich JA. Fused cerebral organoids model interactions between brain regions. Nat. Methods 14(7), 743-751 (2017)

13. Birey F, Andersen J, Makinson CD et al. Assembly of functional forebrain spheroids from human pluripotent cells. Nature 545(7652), 54-59 (2017).

14. Xiang Y, Tanaka Y, Patterson B et al. Fusion of regionally specified hPSC-derived organoids models human brain development and interneuron migration. Cell Stem Cell 21(3), 383-398.e7 (2017).

15. Gabriel E, Ramani Z, Karow $\mathrm{U}$ et al. Recent Zika virus isolates induce premature differentiation of neural progenitors in human brain organoids. Cell Stem Cell. 20(3), 397-406.e5 (2017).

16. Mariani J, Coppola G, Zhng P et al. FOXG1-dependent dysregulation of GABA/glutamate neuron differentiation in autism spectrum disorders. Cell 162(2), 375-390 (2015).

17. Wang P, Mokhtari R, Pedrosa E et al. CRISPR/Cas9-mediated heterozygous knockout of the autism gene CHD8 and characterization of its transcriptional networks in cerebral organoids derived from iPS cells. Mol. Autism 8, 11 (2017).

18. Mellios N, Feldman DA, Sheridan SD et al. MeCP2-regulated miRNAs control early human neurogenesis through differential effects on ERK and AKT signaling. 31(4), 872-881 (2018).

19. Iefremova V, Manikakis G, Krefft O et al. An organoid-based model of cortical development identifies non-cell-autonomous defects in Wnt signaling contributing to Miller-Dieker syndrome. Cell Rep. 19(1), 50-59 (2017).

20. Bershteyn M, Nowakowsky TK, Pollen AA et al. Human iPSC-derived cerebral organoids model cellular features of lissencephaly and reveal prolonged mitosis of outer radial glia. Cell Stem Cell 20(4), 435-449.e4 (2017).

21. Stachowiak EK, Benson CA, Narla ST et al. Cerebral organoids reveal early cortical maldevelopment in schizophrenia - computational anatomy and genomics, role of FGFR1. Transl. Psychiatry 7(11), 6 (2017).

22. Allende ML, Cook EK, Larman BC et al. Cerebral organoids derived from Sandhoff disease-induced pluripotent stem cells exhibit impaired neurodifferentiation. J. Lipid Res. 59(3), 550-563 (2018).

23. Li Y, Mufat J, Ommat A et al. Induction of expansion and folding in human cerebral organoids. Cell Stem Cell 20(3), 385-396.e3 (2017).

24. Watanabe M, Buth JE, Vislaghni N et al. Self-organized cerebral organoids with human-specific features predict effective drugs to combat Zika virus infection. Cell Rep. 21(2), 517-532 (2017).

25. Dang J, Tiwari SK, Lichinchi G et al. Zika virus depletes neural progenitors in human cerebral organoids through activation of the innate immune receptor TLR3. Cell Stem Cell 19(2), 258-265 (2016).

26. Yoon KJ, Song G, Quiang X et al. Zika-virus-encoded NS2A disrupts mammalian cortical neurogenesis by degrading adherens junction proteins. Cell Stem Cell 21(3), 349-358.e6 (2017).

27. Janssens S, Schotsaert M, Karnik R et al. Zika virus alters DNA methylation of neural genes in an organoid model of the developing human brain. mSystems 3(1), e00219-e00317 (2018).

28. Raja WK, Mungenast AE, Lin YT et al. Self-organizing 3D human neural tissue derived from induced pluripotent stem cells recapitulate Alzheimer's disease phenotypes. PLoS ONE 11(9), e0161969 (2016).

29. Lancaster MA. Cerebral organoids model human brain development and microcephaly. Nature 501(7467), 373-379 (2013).

30. Ye F, Kang E, Yu C et al. Erratum: DISC1 regulates neurogenesis via modulating kinetochore attachment of Ndel1/Nde1 during mitosis (Neuron (2017) 96(5) (1041-1054.e5) (S0896627317309789) (10.1016/j.neuron.2017.10.010)). Neuron 96(5), 1204 (2017).

31. Kim YH, Choi SH, Davazo C et al. A 3D human neural cell culture system for modeling Alzheimer's disease. Nat. Protoc. 10(7), 985-1006 (2016)

32. Pham MT, Pollock KM, Rose MD et al. Generation of human vascularized brain organoids. Neuroreport 29(7), 588-593 (2018).

33. Mansour AA, Gonsales JT, Bioyd CW et al. An in vivo model of functional and vascularized human brain organoids. Nat. Biotechnol. 36(5), 432-441 (2018).

34. Bergmann S, Lawler SE, Qu Y et al. Blood-brain-barrier organoids for investigating the permeability of CNS therapeutics. Nat. Protoc. 13(12), 2827-2843 (2018). 